\title{
CIÊNCIANATURA
}

\section{Avaliação da eficiência na atenção básica à saúde no Estado da Paraíba: Uma análise via modelo de regressão beta inflacionado}

\author{
Evaluation of efficiency in basic health care in the Paraíba State: An analysis via inflated \\ beta regression model
}

Camila Ribeiro Silva, Tatiene Correia Souza e Caliandra Maria Bezerra Luna Lima

Universidade Federal da Paraíba, Brasil

\section{Resumo}

Este artigo tem por objetivo identificar os fatores que influenciam a eficiência dos municípios do estado da Paraíba, no que se refere ao gerenciamento dos recursos destinados à saúde, mais especificamente, à atenção básica. Para isso, utilizamos o modelo de regressão beta inflacionado em $(0,1]$ para explicar a eficiência na atenção básica nos municípios paraibanos. Os resultados revelam que, municípios com maior população, melhores condições de saneamento básico e com maior índice FIRJAN de desenvolvimento municipal na saúde, tendem a ser mais eficientes quanto ao gerenciamento de recursos relacionados à atenção básica. Por outro lado, municípios com maior número de consultas médicas por estabelecimento de saúde tendem a ser menos eficientes. A plena eficiência no gerenciamento de recursos da atenção básica, mostrou-se intimamente relacionada ao índice FIRJAN de desenvolvimento municipal de educação.

Palavras-chave: Atenção básica, eficiência, regressão beta inflacionada.

\section{Abstract}

This article aims to identify the factors that influence the efficiency of the municipalities of the state of Paraíba, regarding the management of health resources, more specifically, primary care. For this, we used the inflated beta regression model $(0,1]$ to explain the efficiency in basic care in the municipalities of Paraíba. The results show that municipalities with larger populations, better conditions for basic sanitation and with a higher FIRJAN index of municipal health development, tend to be more efficient in the management of resources related to basic care. On the other hand, municipalities with greater number of medical consultations per health establishment tend to be less efficient. Full efficiency in the management of primary health care resources was shown to be closely related to FIRJAN index of municipal education development.

Keywords: Primary care, efficiency, inflated beta regression. 


\section{Introdução}

A atenção básica $(\mathrm{AB})$ consiste no primeiro nível de assistência sanitária e é responsável pelo conjunto de serviços direcionados às necessidades do indivíduo e a integração dos níveis de atenção à saúde. Ela fundamenta-se em um conjunto de características definidas por Starfield (1998), sendo elas: acessibilidade ao serviço diante de uma primeira necessidade (caracterizando o primeiro contato), o cuidado sendo regular e associado à relação pessoal entre profissionais e pacientes (longitudinalidade), o acesso a toda forma de atenção à saúde (integralidade) e a continuidade da assistência (coordenação), além de ter como foco a família e orientação comunitária, sendo estas duas últimas características, em específico, do Programa de Saúde da Família.

Nos últimos anos a AB tem ganhado abrangência expressiva no país. No final de 2013, haviam 36 mil equipes de Estratégia de Saúde da Família, 300 mil agentes comunitários de saúde e 23 mil equipes de saúde bucal (Rodrigues et al., 2014). No entanto, apesar desses avanços, seu financiamento ainda tem sido controverso, pois ainda apresenta insuficiência (Mendes e Marques, 2014). A importância dada a esse nível de atenção pode ser explicada pelo fato de que um sistema eficiente em cuidados primários, proporciona melhorias substanciais de saúde, com custos relativamente pequenos, principalmente quando comparados aos níveis secundário e terciário, que compreendem a assistência prestada pelas unidades de pronto atendimento e hospitais escolas, e a assistência de hospitais de grande porte, respectivamente.

É possível verificar, através de ampla literatura, os inúmeros benefícios que um sistema de AB pode promover, estando bem estruturado e interligado com os níveis superiores (Engstrom et al., 2001). Nesses benefícios estão incluídos melhores resultados de saúde (Forrest e Starfield, 1996; Starfield, 1998), atenção maior na prevenção e na detecção precoce de problemas de saúde (Shea et al., 1992), redução de mortalidade por todas as causas (Shi, 1992; Villalbi et al., 1999), reduz as re-hospitalizações (Weinberger et al., 1996), as consultas com especialistas (Woodward et al., 2004), induz um menor uso de serviços de emergência e melhora a detecção de fatores adversos de intervenções médicas (Kravitz et al., 2004).

Através da avaliação do desempenho municipal é possível mensurar a situação dos serviços de saúde no âmbito da atenção básica. A eficiência em saúde pode ser compreendida como a relação entre o custo e o impacto dos serviços sobre a saúde da população, preservado um nível de qualidade determinado (Viacava et al., 2012). Conforme Donabedian (2003), a eficiência em saúde representa a capacidade de um sistema de funcionar com menores custos sem prejudicar os resultados esperados, com o objetivo de atingir a melhor relação de custo benefício. Diante dos conceitos do que seria eficiência em saúde, alguns trabalhos nos últimos anos buscaram mensurá-la e descrevê-la como um mecanismo para uma gestão de recursos mais eficaz. Entretanto, ainda são poucos os estudos que buscaram avaliar a eficiência em saúde no Brasil. Alguns trabalhos se destinaram a avaliar as ações, procedimentos e serviços de saúde específicos no contexto da média e alta complexidade.

No âmbito do SUS, a média e alta complexidade é definida pela totalidade de procedimentos que envolvem alta tecnologia e alto custo, tendo por objetivo principal disponibilizar a população serviços especializados e qualificados (BRASIL, 2007). Dessa forma, o custo operacional demandado com esses níveis de atenção são maiores, e por isso, mensurar a eficiência em saúde torna-se uma importante ferramenta de avaliação. Especificamente relacionado à AB, foram encontrados os estudos de Varela et al. (2012) e Alves (2012), que limitaram-se aos estados de São Paulo e Espírito Santo, respectivamente, e Dias (2010) que realizou um estudo voltado aos municípios brasileiros.

Ademais, outros estudos avaliaram a eficiência na aplicação de recursos públicos municipais e buscaram identificar os impactos sobre os indicadores de atenção à saúde (Varela et al., 2012; Faria et al., 2008; Marinho, 2003). Conforme Varela et al. (2012), que realizou um estudo com municípios paulistas, seria possível aumentar, expressivamente, a quantidade de serviços de saúde prestados à população sem a necessidade de novos custos, na maioria dos municípios. Faria et al. (2008) avaliou a eficiência dos gastos municipais em saúde e educação no estado do Rio de Janeiro, e ressalta que a eficiência não está relacionada à disponibilidade maior ou menor de recursos. Pois, é possível que um município gaste pouco, porém, invista esses recursos de forma eficiente, enquanto um município gaste muito e aplique os seus recursos de forma inadequada. Apesar de iniciativas para aprimorar o financiamento da $\mathrm{AB}$, sabe-se que os municípios apresentam diversos tipos de dificuldades, em particular, os municípios menores que não contam com recursos próprios suficientes para financiar as despesas não cobertas pelo governo federal (Mendes e Marques, 2014).

Neste sentido, o objetivo do presente artigo é identificar os fatores que influenciam a eficiência dos municípios da Paraíba, no que se refere ao gerenciamento dos recursos destinados à $\mathrm{AB}$. Como a variável de interesse possui valores limitados ao intervalo $(0,1]$, os modelos lineares usuais tornam-se inadequados. Assim, faz-se necessário o uso de modelos mais flexíveis para situações em que a variável de interesse é taxa ou proporção. Dessa forma, foi utilizado o modelo de regressão beta inflacionado proposto por Ospina e Ferrari (2012).

O presente artigo encontra-se dividido em quatro seções, incluindo esta introdução. Na Seção 2 é apresentado o modelo de regressão beta inflacionado. Uma breve descrição da base de dados utilizada e resultados obtidos são apresentados na Seção 3. Por fim, algumas conclusões e comentários são apresentados na Seção 4. 


\section{Modelo de regressão beta inflacionado}

Modelos de regressão constituem-se em ferramentas úteis para resumir e interpretar dados. Em muitas áreas do conhecimento empregam-se modelos de regressão para analisar a relação entre variáveis (Dias, 2010; Sousa, 2011; Souza et al., 2016). A análise de regressão convencional assume a suposição de que os erros têm distribuição normal e geralmente é a mais utilizada em várias aplicações. No entanto, este modelo torna-se inadequado quando a variável de interesse esta restrita a um intervalo, a exemplo de taxas ou proporções. Sendo a variável limitada, geralmente, ao intervalo $(0,1)$, os valores estimados para a variável de interesse a partir da modelagem clássica de regressão podem, por exemplo, exceder os limites da variável resposta, entre outras violações do modelo.

Uma alternativa utilizada para esse problema é aplicar uma transformação na variável dependente (resposta) de forma que ela possa assumir valores na reta real. No entanto, uma desvantagem está na perda de interpretabilidade dos parâmetros, visto que não serão interpretados em termos da resposta original (Paolino, 2001), dependendo da transformação. Além disso, distribuições de variáveis do tipo taxa ou proporção são tipicamente assimétricas, e desse modo, realizar inferências na suposição de normalidade pode levar a conclusões erradas.

Ferrari e Cribari-Neto (2004) propuseram uma classe de modelos de regressão beta em que a resposta média é relacionada a um preditor linear, que envolve covariáveis e parâmetros de regressão desconhecidos, através de uma função de ligação. O modelo também é composto por um parâmetro de precisão e supõe que a variável dependente tem distribuição beta. A classe de modelos de regressão beta é usada para modelar dados que assumem valores no intervalo unitário padrão $(0,1)$ citepsilva,almeida,cribarisouza. Trata-se de um modelo heteroscedástico e que facilmente acomoda assimetrias. Simas et al. (2010) apresentaram uma variante do modelo de regressão beta que introduz não-linearidade e dispersão variável.

Ospina e Ferrari (2012) introduziram uma classe de modelos de regressão beta inflacionados em que a resposta segue uma distribuição beta inflacionada, os quais são uma extensão natural do modelo de regressão beta. O modelo inclui um submodelo de regressão para a probabilidade de que a variável dependente seja igual a um dos limites do intervalo. A parte contínua dos dados é modelada através da distribuição beta e a parte discreta, ou seja, o ponto de massa, é modelada a partir de uma distribuição degenerada no valor conhecido $c$ (em que $c$ é igual a 0 ou 1) e o parâmetro de precisão satisfaz algumas relações funcionais, ver Cribari-Neto e Pereira (2013).

Sejam $y_{1}, \ldots, y_{n}$, variáveis aleatórias independentes, em que cada $y_{t}, t=1, \ldots n$, segue a função de densidade Ospina e Ferrari (2010)

$$
b i_{c}= \begin{cases}\alpha_{t}, & y=c, \\ \left(1-\alpha_{t}\right) f\left(y ; \mu_{t}, \phi_{t}\right), & y \in(0,1),\end{cases}
$$

em que $0<\alpha_{t}<1$ é o parâmetro de mistura da distribuição, dado por $\alpha_{t}=\operatorname{Pr}\left(y_{t}=c\right), 0<\mu_{t}<1$ é a média de $y_{t}$ condicionada em $y_{t} \in(0,1), \phi_{t}>0$ é o parâmetro de precisão e a $f\left(y ; \mu_{t}, \phi_{t}\right)$ é a função de densidade beta.

Sob a parametrização de Ferrari e Cribari-Neto (2004), a densidade beta é dada por

$$
f\left(y_{t} ; \mu_{t}, \phi_{t}\right)=\frac{\Gamma\left(\phi_{t}\right)}{\Gamma\left(\mu_{t} \phi_{t}\right) \Gamma\left(\left(1-\mu_{t}\right) \phi_{t}\right)} y_{t}^{\left(\mu_{t} \phi_{t}-1\right)}\left(1-y_{t}\right)^{\left(1-\mu_{t}\right) \phi_{t}-1},
$$

em que $0<y_{t}<1,0<\mu_{t}<1$, e $\phi_{t}>0$.

A densidade (1) é de uma variável aleatória beta inflacionada no ponto $c, c=0$ ou $c=1$. Se $c=0$, dizemos que a distribuição é beta inflacionada no ponto zero. Se $c=1$, dizemos que a distribuição é beta inflacionada no ponto um. Para esta distribuição temos que $\mathrm{E}\left(y_{t}\right)=\alpha_{t} c+\left(1-\alpha_{t}\right) \mu_{t}$ e $\operatorname{Var}\left(y_{t}\right)=\left(1-\alpha_{t}\right) \mu_{t}\left(1-\mu_{t}\right) /\left(\phi_{t}+1\right)+\alpha_{t}\left(1-\alpha_{t}\right)\left(c-\mu_{t}\right)^{2}$.

O modelo de regressão beta inflacionado em $c$ com dispersão variável é definido supondo que a média condicional de $y_{t}$, a massa de probabilidade em $c$ e o parâmetro de precisão satisfazem às seguintes relações funcionais:

$$
\begin{aligned}
& h\left(\alpha_{t}\right)=\sum_{i=1}^{M} z_{t i} \gamma_{i}=\zeta_{t}, \\
& g\left(\mu_{t}\right)=\sum_{i=1}^{m} x_{t i} \beta_{i}=\eta_{t}, \\
& b\left(\phi_{t}\right)=\sum_{i=1}^{q} s_{t i} \lambda_{i}=k_{t},
\end{aligned}
$$


em que $\gamma=\left(\gamma_{1}, \ldots, \gamma_{M}\right)^{\top}, \beta=\left(\beta_{1}, \ldots, \beta_{m}\right)^{\top}$ e $\lambda=\left(\lambda_{1}, \ldots, \lambda_{q}\right)^{\top}$ são vetores de parâmetros de regressão desconhecidos, tais que $\gamma \in \mathbb{R}^{M}, \beta \in \mathbb{R}^{m}, \lambda \in \mathbb{R}^{q}, z_{t 1}, \ldots, z_{t M}, x_{t 1}, \ldots, x_{t m}$, e $s_{t 1}, \ldots, s_{t q}$ são observações de variáveis conhecidas $(m+M+q<n)$ que podem coincidir total ou parcialmente e $\zeta_{t}, \eta_{t}$ e $k_{t}$ são os preditores lineares. As funções de ligação $h(\cdot)$, $g(\cdot)$ e $b(\cdot)$ são estritamente monótonas e duas vezes diferenciável.

No modelo de regressão beta inflacionado, os parâmetros $\alpha_{t}, \mu_{t}$ e $\phi_{t}$ são definidos como funções lineares de $\gamma, \beta$ e $\lambda$, respectivamente, através das relações apresentadas em 2,3 e 4 , isto é, $\alpha_{t}=h^{-1}\left(\zeta_{t}\right), \mu_{t}=g^{-1}\left(\eta_{t}\right)$ e $\phi_{t}=b^{-1}\left(k_{t}\right)$. Entre as funções de ligação mais utilizadas para $\mu$ e $\alpha$ estão a função de ligação logit com $g(\mu)=\log (\mu /(1-\mu))$, a função probit com $g(\mu)=\Phi^{-1}(\mu)$, em que $\Phi(\cdot)$ é a função de distribuição normal padrão, a log-log complementar com $g(\mu)=\log (-\log (1-\mu))$, a ligação $\log \log \operatorname{com} g(\mu)=-\log (-\log (\mu))$ e a função de ligação Cauchy com $g(\mu)=\tan (\pi(\mu-0.5))$. Por outro lado, para o parâmetro de precisão $\phi$, é possível utilizar a função logarítmica, com $b(\phi)=\log (\phi)$ ou raiz quadrada $b(\phi)=\sqrt{\phi}$. Para mais detalhes sobre estas funções ver McCullagh e Nelder (1989).

A estimação dos parâmetros é realizada através do método de máxima verossimilhança, no entanto, como os estimadores não possuem forma fechada eles precisam ser obtidos numericamente maximizando a função de log-verossimilhança, através de um algoritmo de maximização não-linear. Usualmente, utiliza-se o método quasi-Newton BFGS (Press et al., 1992). Para maiores detalhes sobre aspectos inferenciais e expressões matriciais do vetor escore e matriz de informação de Fisher, ver Ospina e Ferrari (2012) e Pereira e Cribari-Neto (2014). O procedimento computacional foi desenvolvido utilizando o pacote GAMLSS (Modelos Aditivos Generalizados para Locação, Escala e Forma) (Stasinopoulos e Rigby, 2007) do software estatístico R (R Core Team, 2013; Cribari-Neto e Zeileis, 2010).

\subsection{Adequabilidade do modelo}

Para avaliar a correta especificação do modelo ajustado foi aplicado o teste de especificação RESET (Ramsey, 1969; Pereira e Cribari-Neto, 2014). A hipótese nula do teste consiste de que o modelo está bem especificado, versus a hipótese alternativa de má especificação. Essa má especificação do modelo podendo ser decorrente de variável regressora omitida, não linearidade negligenciada ou forma distribucional incorreta. Ademais, também foi utilizado o teste de Moran (Cliff e Ord, 1973) para medir a associação espacial entre os municípios, tendo em vista que municípios vizinhos tendem a ter características semelhantes, em geral. Em conjunto com o teste de Moran utilizou-se o teste de permutação da estatística de Moran (Cliff e Ord, 1981). Para a avaliação da adequabilidade do modelo utilizou-se o coeficiente de determinação (pseudo- $R^{2}$ ) de McFadden (1974).

Após a obtenção do modelo, uma etapa importante é a verificação de possíveis afastamentos das suposições assumidas para o modelo. Neste sentido, é importante a detecção de observações que podem exercer efeito desproporcional no ajuste e influenciar nos resultados inferenciais, em alguns casos. Para tanto, foram considerados os gráficos dos resíduos quantis aleatorizados versus os índices das observações e o gráfico de probabilidade normal com envelopes simulados. Técnicas gráficas utilizando resíduos são frequentemente adotadas para análise de diagnóstico (Silva, 2015; Pereira et al., 2014).

\section{Resultados e discussão}

A base de dados utilizada faz parte de um trabalho realizado por Sousa (2011), que buscou computar índices de eficiência para a AB nos municípios brasileiros. Para tanto, a autora utilizou a metodologia não-paramétrica DEA (Análise Envoltória de Dados) (Charnes et al., 1978) para construção dos escores de eficiência. Para cada município observado foi atribuído um valor que representa a eficiência técnica na aplicação dos recursos destinados à $\mathrm{AB}$. Os municípios que apresentam escore de eficiência igual a 1 (um), são denominados de plenamente eficientes. A amostra foi obtida considerando um corte seccional para o ano de 2007, com exceção apenas das variáveis PCI e PODER, que referem-se ao ano de 2000. A amostra utilizada no presente artigo refere-se ao estado da Paraíba, no total de 154 municípios observados. A base de dados disponibilizada também contém informações de variáveis socioeconômicas e relacionadas à saúde, que foram coletadas em fontes de domínio público como IBGE (Instituto Brasileiro de Geografia e Estatística), DATASUS (Departamento de Informática do Sistema Único de Saúde), FIRJAN (Federação das Indústrias do estado do Rio de Janeiro), SIAB (Sistema de informação de atenção básica) e CNES (Cadastro Nacional de Estabelecimentos de saúde). Os escores de eficiência baseados no modelo DEA foram considerados como variável dependente, enquanto as demais variáveis (15 no total) foram consideradas como explicativas, sendo descritas na Tabela 1.

Na Tabela 2 são apresentadas estatísticas descritivas das variáveis contínuas e a frequência para as variáveis dummy utilizadas no ajuste. É possível verificar que o valor máximo e mínimo para a variável EFIC nos municípios foi de 1,000 e 0,3512, respectivamente; e mediana igual a 0,6536. Além disso, verificamos que a eficiência média dos municípios foi de 0,6867, com desvio padrão (DP) igual a 0,1769. Apenas 9\% dos municípios paraibanos configuram-se como plenamente eficientes e $14,9 \%$ apresentam eficiência abaixo de 0,5. Nota-se que a população (POP) média nos municípios foi de 20.118 habitantes, a média de famílias com cobertura dos serviços de saneamento básico (SANEA) foi de 0,4182 com desvio padrão de 0,1774. Os valores mínimo e máximo para a variável SANEA foram 0,0231 e 0,9258, respectivamente.

O índice FIRJAN de Desenvolvimento Municipal é um estudo realizado pelo sistema FIRJAN, que acompanha anualmente o desenvolvimento socioeconômico dos municípios brasileiros, em três áreas de atuação: emprego e renda, educação e saúde. 
Tabela 1: Descrição das variáveis utilizadas no modelo de regressão beta inflacionado.

\begin{tabular}{ll}
\hline \hline Variável & Definição \\
\hline EFIC & Escores de eficiência para a tenção básica. \\
PCI & Dummy: 1 se o município participa de consórcio intermunicipal, 0 caso contrário. \\
PODER & Dummy: 1 se o conselho municipal tem poder de decisão, 0 caso contrário. \\
POP & População do município. \\
IFDMR & Indice FIRJAN de desenvolvimento municipal - componente renda. \\
IFDME & Indice FIRJAN de desenvolvimento municipal - componente educação. \\
IFDMS & Indice FIRJAN de desenvolvimento municipal - componente saúde. \\
MED-ENF & Relação entre o número de médicos e enfermeiros da atenção básica municipal. \\
CAP & Dummy: igual a 1 se o município for a capital do estado, 0 caso contrário. \\
SANEA & Média dos percentuais de famílias atendidas pelas equipes de saúde da família \\
& que possuem água em rede, esgoto e coleta de lixo. \\
IDOS & Proporção de idosos no município em 2007. \\
INFANT & Proporção de crianças no município em 2007. \\
CONSULT & Número de consultas médicas por estabelecimento de saúde. \\
PROF & Média de médicos por estabelecimento da atenção básica. \\
HOSP & Dummy: igual a 1 se o município possui hospital, 0 caso contrário. \\
REG & Dummy: igual a um se o município pertence a uma região metropolitana, 0 caso contrário. \\
\hline \hline
\end{tabular}

Desenvolvido em 2008, o índice é baseado, exclusivamente, em estatísticas públicas oficiais, disponibilizadas pelos ministérios do Trabalho, Educação e Saúde. O índice varia de 0 (mínimo) a 1 (máximo), em que quanto mais próximo de 1, maior o nível de desenvolvimento do município. Com relação aos índices FIRJAN (FIRJAN, 2015), observa-se que o índice médio para a componente saúde foi de 0,6811 , o índice médio na renda de 0,3073 e o índice médio na educação sendo de 0,5755 . Além disso, a média de consultas por unidade de saúde (CONSULT) foi de 3.489,76 consultas, com número mínimo de 327 e máximo de 9.602 consultas, respectivamente.

A proporção média de crianças (INFANT) e idosos (IDOS) nos municípios foi de 0,0953 e 0,1183, respectivamente. Conforme a análise verifica-se que apenas 21,43\% dos municípios têm poder de decisão (PODER) através dos conselhos municipais, 22,73\% participam de consórcio intermunicipal, 6,49\% pertencem a região metropolitana e que apenas 41,56\% deles possui hospital Nota-se que os desvios-padrão (DP) das variáveis observadas foram, em sua maioria, relativamente baixos, indicando menor dispersão em torno da média. Além disso, as maiores variações em torno da média, expressa pelo coeficiente de variação (CV), foram apresentadas pelas variáveis POP e CONSULT, com valores de 3,1721 e 0,4984, respectivamente.

Ao analisarmos as correlações entre às variáveis contínuas foi possível verificar que essas foram consideradas baixas $(<0,40)$, de modo geral. Exceto para as variáveis POP e IFDMR, que apresentaram uma correlação positiva de valor igual a 0,6403, e para as variáveis PROF e CONSULT, que também se mostraram correlacionadas positivamente $(0,4159)$, isto é, quanto maior o número de profissionais médicos por unidade de saúde, maior será o número de consultas realizadas. De acordo com as análises foi observado que o município de Sobrado apresentou o menor escore de eficiência entre os municípios, sendo de 0,3512. Em contrapartida, os municípios plenamente eficientes foram: Aguiar, Algodão de Jandaíra, Amparo, Areia de Baraúnas, Belém do Brejo do Cruz, Guarabira, Riachão, Poço de José de Moura, Quixabá, Riacho de Santo Antônio, São Francisco, São José dos Cordeiros, Serra Grande e Zabelê. Para esses municípios foi observado que alguns apresentaram em comum altos índices FIRJAN de desenvolvimento na saúde, baixos índices FIRJAN de desenvolvimento na renda e elevado número de consultas por estabelecimento.

Com relação a população municipal, o valor máximo e mínimo para essa variável foram observados nos municípios de João Pessoa e Quixabá, com 683.278 e 1.004 habitantes, respectivamente. O menor percentual de famílias com serviços de saneamento básico foi registrado no município de Juazeirinho (0,0231), e o maior percentual no município Poço de José de Moura (0,9258). Quanto ao número de consultas por unidade de saúde, o município de Amparo se destacou entre os demais com 9.602 consultas, enquanto o município de Riachão foi o que apresentou o menor número (327). Para o índice FIRJAN de desenvolvimento municipal na educação, o município de Várzea foi o que apresentou maior índice, com valor de 0,7743, sobrepondo-se ao valor observado na capital do estado de 0,6276. Enquanto São Bento foi o município com menor índice de desenvolvimento na educação $(0,4316)$. Quando avaliamos esse mesmo índice considerando a componente saúde, verificamos que o município de Santarém apresentou o maior índice, sendo de 0,9051, e Curral Velho o menor (0,4781). Para a capital do estado, o índice de desenvolvimento municipal na saúde foi de 0,7914 .

A plena eficiência quanto ao gerenciamento dos recursos destinados à atenção básica no estado foi predominante nos municípios menores, com menos de cinco mil habitantes (IBGE, 2017), com exceção apenas das cidades de Aguiar, Belém do Brejo do Cruz e Guarabira, com populações de 5.562, 7.347 e 58.881 habitantes, respectivamente. Esse resultado corrobora com o estudo realizado por Queiroz et al. (2013) em cidades do Rio Grande do Norte, em que a plena eficiência no gasto público em saúde foi expressa 
Tabela 2: Medidas descritivas das variáveis contínuas e frequência das variáveis dummy.

\begin{tabular}{lccccccc}
\hline \hline Variável & mínimo & média & mediana & máximo & DP & CV & Frequência (\%) \\
\hline EFIC & 0,3512 & 0,6867 & 0,6536 & 1,0000 & 0,1769 & 0,2576 & - \\
POP & 1.004 & 20.118 & $8.375,50$ & 683.278 & $63.815,61$ & 3,1721 & - \\
SANEA & 0,0231 & 0,4182 & 0,3883 & 0,9258 & 0,1774 & 0,4242 & - \\
IFDMS & 0,4789 & 0,6811 & 0,6787 & 0,9051 & 0,0841 & 0,1235 & - \\
IFDMR & 0,0458 & 0,3073 & 0,2919 & 0,8630 & 0,1049 & 0,3412 & - \\
IFDME & 0,4316 & 0,5755 & 0,5704 & 0,7743 & 0,0592 & 0,1028 & - \\
CONSULT & 327 & $3.489,76$ & $3.250,33$ & 9.602 & $1.739,33$ & 0,4984 & - \\
ME-ENF & 0,5500 & 1,0897 & 1,0000 & 3,0000 & 0,2591 & 0,2378 & - \\
INFANT & 0,0677 & 0,0953 & 0,0951 & 0,1340 & 0,0123 & 0,1296 & - \\
IDOS & 0,0499 & 0,1183 & 0,1193 & 0,1705 & 0,0231 & 0,1952 & - \\
PROF & 0,2000 & 1,1347 & 1,0000 & 3,0000 & 0,4790 & 0,4222 & - \\
PODER & - & - & - & - & - & - & $33(21,43)$ \\
PCI & - & - & - & - & - & - & $35(22,73)$ \\
CAP & - & - & - & - & - & - & 1 \\
REG & - & - & - & - & - & - & $10(6,49)$ \\
HOSP & - & - & - & - & - & - & $64(41,56)$ \\
\hline \hline
\end{tabular}

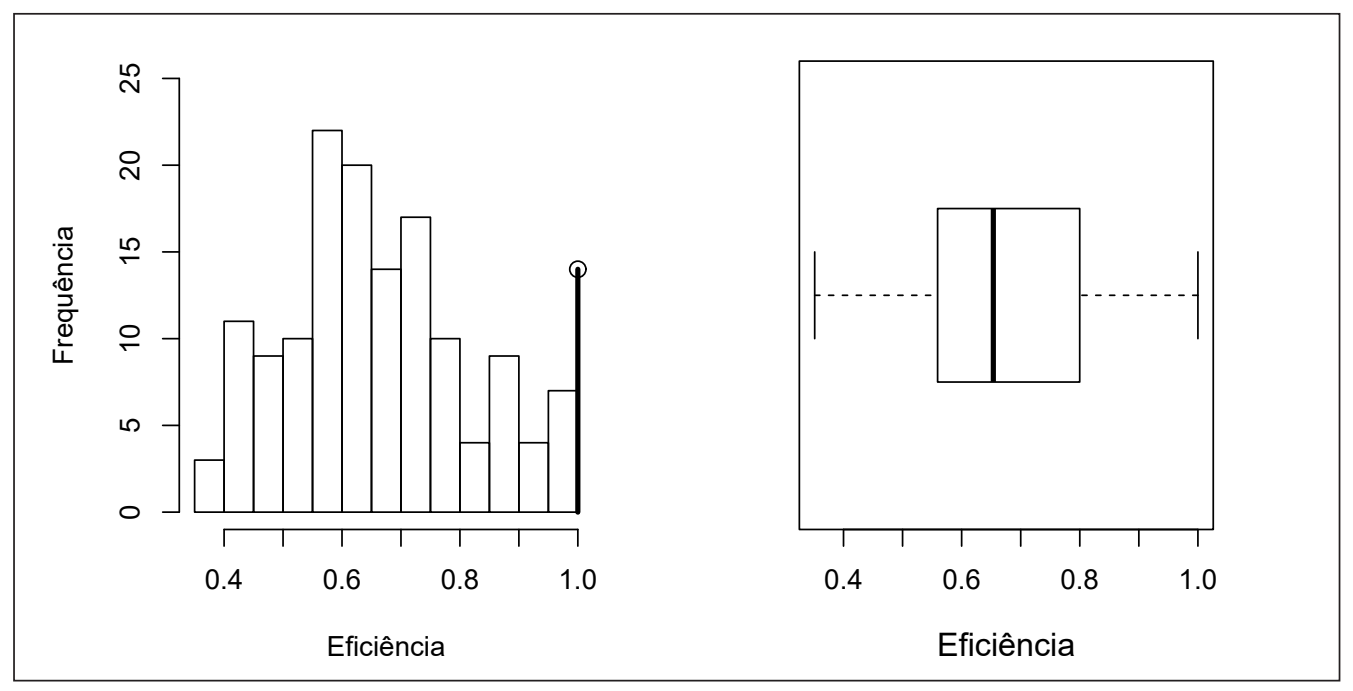

Figura 1: Histograma de frequências e box-plot da variável Eficiência.

por municípios de grande e pequeno porte populacional. Por outro lado, em Varela et al. (2012) que analisou municípios paulistas, o grupo de cidades ineficientes era predominantemente formado por municípios de pequeno porte populacional. Desse modo, é importante salientar que não apenas o porte do município irá definir sua capacidade de ser ou não plenamente eficiente. $\mathrm{O}$ achado no presente estudo contribui para uma melhor compreensão da situação de assistência à saúde prestada à população, bem como o nível de gerenciamento dos recursos da $\mathrm{AB}$ no respectivo município. Uma vez que, os resultados podem diferir segundo a região analisada, conforme observado ao se comparar os resultados do presente trabalho com os apresentados por Varela et al. (2012).

Na Figura 1 apresentamos o histograma e o box-plot da variável EFIC. Observa-se que a distribuição da variável em $(0,1]$ é assimétrica, apresentando coeficiente de assimetria de Pearson igual a 0,558. Além disso, a partir do box-plot, nota-se que a mediana está mais próxima do primeiro quartil.

\subsection{Especificação do modelo}

Com relação ao ajuste do modelo, inicialmente o interesse foi de verificar a hipótese nula de que o parâmetro de precisão do modelo é fixo, ou seja, se há ou não estrutura de regressão para o parâmetro de precisão. Para tanto, utilizamos o teste da razão de verossimilhança (Cribari-Neto e Pereira, 2013), com hipótese nula de que a precisão é constante, isto é, $\mathcal{H}_{0}: \phi_{1}=\ldots=\phi_{n}=\phi$, versus a hipótese alternativa de que $\mathcal{H}_{1}: \phi_{i} \neq \phi_{j}$, para algum $i \neq j$. Rejeitamos ao nível de $5 \%$ a hipótese nula de precisão fixa 
( $p$-valor $\left.<3,803 \times 10^{-8}\right)$. Desse modo, podemos concluir que a precisão é variável para o modelo de regressão beta inflacionado. Logo, além de modelarmos a média condicional e a massa de probabilidade em um, também será necessário modelar o parâmetro de precisão do modelo. As funções de ligação utilizadas para o modelo foram logit, logit e log, para a média condicional, massa de probabilidade em um e precisão, respectivamente. Vale salientar que foram testadas outras funções de ligação, entretanto, com essas foram encontrados os melhores ajustes.

Para avaliar a relação de dependência espacial entre os municípios, aplicamos o teste de Moran. O $p$-valor obtido foi de 0,4562, desse modo, ao nível de significância de 5\% não rejeitamos a hipótese nula de independência entre os municípios. Ou seja, não existe evidência estatística de dependência entre os municípios paraibanos quanto a eficiência no gerenciamento de recursos destinados à $\mathrm{AB}$. Além disso, pelo teste de permutação da estatística de Moran, também foi apontada a independência entre os municípios ( $p$-valor=0,45). Uma possível explicação para isso pode estar no fato da AB ter territorialização. Isto é, todos os municípios têm sua área de atuação e população definida perante os serviços direcionados à $\mathrm{AB}$. Com a territorialização, os serviços oferecidos variam de acordo com a situação de saúde de cada população, existindo de certo modo, autonomia entre essas unidades.

Com o intuito de verificar possíveis afastamentos das suposições adotadas para o modelo, a Figura 2 apresenta os gráficos dos resíduos quantis aleatorizados versus os índices das observações e o gráfico de probabilidade normal com envelopes simulados. $\mathrm{O}$ modelo de regressão parece estar bem ajustado, uma vez que os resíduos encontram-se dentro do intervalo $(-3,3)$. Além disso, pode-se verificar que os resíduos permanecem dentro das bandas de confiança dos envelopes simulados, em geral, não havendo, portanto, indícios de afastamento da suposição de que o modelo de regressão beta inflacionado fornece uma boa representação para os dados observados.

O teste RESET foi realizado utilizando o quadrado do preditor linear estimado como variável de teste, uma vez que estudos de simulação evidenciaram uma melhor performance em termos de tamanho e poder quando utilizada a segunda potência do preditor linear como variável de teste (Pereira e Cribari-Neto, 2014). O p-valor do teste foi de 0,191, indicando que não rejeitamos ao nível de significância de 5\% a hipótese nula de que o modelo encontra-se bem especificado. Com relação à adequabilidade do modelo, medida através do pseudo- $R^{2}$, o valor obtido foi de 0,849 , indicando uma boa qualidade no ajuste do modelo.

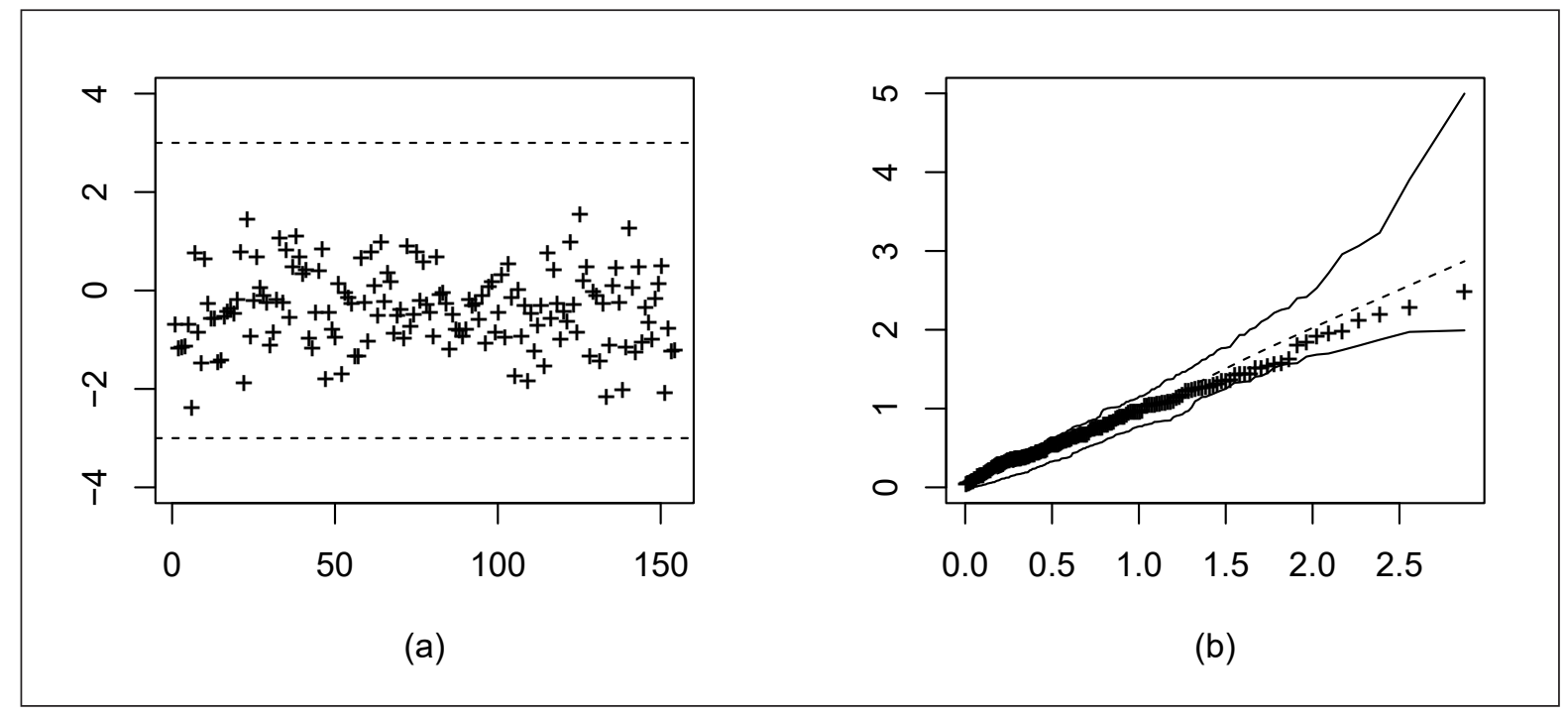

Figura 2: Gráfico dos resíduos quantis aleatorizados versus os índices das observações (a) e gráfico de probabilidade normal com envelopes simulados (b).

Através da análise dos coeficientes estimados para o modelo (Tabela3), é possível verificar que municípios com maior população (POP), melhores condições de saneamento básico (SANEA) e com maior índice FIRJAN de desenvolvimento municipal na saúde (IFDMS), tendem a ser mais eficientes, isto é, tendem a apresentar maior escore de eficiência. O que faz sentido, visto que um sistema de saneamento básico em boas condições pode proporcionar a prevenção de inúmeras doenças (FUNASA, 2006). Em contra partida, o sinal negativo da estimativa para a variável CONSULT, indica que quanto maior o número de consultas médicas por estabelecimento de saúde, menor será a eficiência média do município. Esse resultado podendo ser representado pela superlotação nas unidades básicas de saúde e falta de profissionais em número suficiente para a demanda de cada localidade. Esses resultados confirmam as conclusões realizadas por Dias (2010), em estudo sobre a eficiência da atenção básica nos municípios brasileiros. Através da análise verificou-se que as variáveis IFDMS, as condições de saneamento básico e a população influenciam no aumento do escore de eficiência do município.

Em relação à estrutura de regressão para a massa de probabilidade em um, tem-se que apenas a variável índice FIRJAN de 
desenvolvimento municipal na éducação (IFDME) foi significativa ao nível de 5\%. Assim, a eficiência plena dos municípios paraibanos mostra-se intimamente relacionada ao grau de desenvolvimento do município na educação. O nível de escolaridade do indivíduo é considerado um elemento essencial a ser considerado tanto na análise dos determinantes da saúde, bem como na abordagem da população para o avanço de práticas de promoção, prevenção e recuperação da saúde. O baixo grau de escolaridade pode afetar negativamente no desenvolvimento de conceitos de autocuidado em saúde, na conservação ambiental e na percepção de agir do indivíduo enquanto cidadão nos contextos sanitários coletivos (BRASIL, 2004).

Quanto à estrutura de regressão para a precisão, verifica-se que os municípios com maior índice FIRJAN de desenvolvimento municipal na renda e maior número de consultas, tendem a apresentar respostas mais precisas para a eficiência, assim como a capital do estado. Esse resultado pode ser justificado pelo fato das capitais apresentarem, por exemplo, a disponibilização de mais recursos, mão de obra mais especializada e alocação mais efetiva de recursos, possibilitando maior abrangência no atendimento. Além disso, cidades com melhores índices de desenvolvimento na renda, podem apresentar menor nível de desigualdade no aspecto econômico.

Por outro lado, municípios com maior proporção de idosos e maior média de médicos por estabelecimento de saúde, tendem a ter respostas menos precisas para a eficiência. No âmbito da AB, enfermeiros capacitados podem realizar alguns procedimentos que são realizados pelos médicos a um custo inferior, permitindo, assim, o uso mais eficiente dos recursos públicos (Greenfield et al., 1978). Além disso, o envelhecimento populacional, traz consigo a maior propensão de doenças crônicas (Duncan et al., 2012) que, em geral, conduzem a tratamentos contínuos e dispendiosos. Neste sentido, as unidades municipais apresentam diferentes desempenhos quanto ao enfrentamento dos problemas de saúde desta parcela da população, fazendo com que os escores de eficiência sejam mais dispersos, para esses municípios.

Tabela 3: Modelo de regressão beta inflacionado ajustado para o estado da Paraíba.

\begin{tabular}{lccc}
\hline \hline$\mu$ & & & \\
\hline Variável & Estimativa & Erro-padrão & $\mathrm{p}$-valor \\
\hline Intercepto & 0,5865 & 0,4879 & 0,2313 \\
POP & $9,758 \times 10^{-7}$ & $2,428 \times 10^{-7}$ & $<0,001$ \\
SANEA & 0,8336 & 0,3070 & 0,0074 \\
IFDMS & 1,6650 & 0,6854 & 0,0163 \\
CONSULT & $-7,410 \times 10^{-5}$ & $2,976 \times 10^{-5}$ & 0,0139 \\
\hline$\phi$ & & & \\
\hline Intercepto & 2,5050 & 0,8370 & 0,0032 \\
IFDMR & 2,8870 & 1,2030 & 0,0177 \\
CAPITAL & 1,4800 & 0,7815 & $<0,001$ \\
IDOS & $-1,2710$ & 5,2160 & 0,0160 \\
CONSULT & $2,392 \times 10^{-4}$ & $7,847 \times 10^{-5}$ & 0,0027 \\
PROF & $-0,5710$ & 0,2516 & 0,0247 \\
\hline$\alpha$ & \multicolumn{3}{l}{} \\
\hline Intercepto & $-7,8620$ & 2,7440 & 0,0048 \\
IFDME & 9,4350 & 4,5290 & 0,0389 \\
\hline \hline
\end{tabular}

\section{Conclusões}

Neste artigo avaliamos os fatores que influenciam a eficiência no gasto público em saúde no estado da Paraíba, através do modelo de regressão beta inflacionado. De acordo com os resultados apenas $9 \%$ dos municípios foram considerados plenamente eficientes na aplicação dos recursos destinados à atenção básica e aproximadamente $15 \%$ apresentaram escore de eficiência abaixo de 0,5 , na amostra observada (154 municípios). Ressaltamos que a utilização do modelo de regressão beta inflacionado é adequada pela ocorrência de plena eficiência em alguns municípios da Paraíba e, além disso, o fato de aproximadamente $15 \%$ dos municípios observados apresentarem eficiência abaixo de 0,5 , indica que essa variável possui assimetria, o que foi verificado através do histograma e coeficiente de assimetria.

$\mathrm{Na}$ análise descritiva, verificamos que a proporção média de crianças e idosos é relativamente baixa (menos de $12 \%)$ nos municípios observados. Nota-se também o número reduzido de municípios com hospitais, sendo de apenas 64 entre os municípios observados. Isso faz com que as unidades municipais que não dispõe de serviços de assistência médico-hospitalar, recorram aos municípios mais próximos em busca de assistência, causando, muitas vezes, superlotação dessas unidades. Em apenas $22,43 \%$ dos municípios o conselho municipal tem poder de decisão no processo sobre a política de saúde municipal. Essa participação 
configura-se como um mecanismo de facilitação para a gestão pública no âmbito da saúde e, portanto, sendo importante uma maior abrangência de municípios.

A plena eficiência na aplicação dos recursos destinados à atenção básica foi predominante em municípios menores (com menos de cinco mil habitantes). Essa informação pode ser útil na orientação de futuros planejamentos com relação à políticas públicas voltadas para a assistência à saúde, uma vez que esses municípios podem ter desenvolvido ações, serviços ou programas que podem ter ocasionando um maior escore de eficiência.

No ajuste do modelo de regressão beta inflacionado observamos que a população do município, condições de saneamento básico e índice FIRJAN de desenvolvimento municipal na saúde, são fatores que influenciam positivamente a eficiência média dos municípios, ou seja, são variáveis que estão relacionadas ao aumento do escore de eficiência. Por outro lado, o número de consultas médicas por estabelecimento de saúde exerce efeito negativo sobre a eficiência média do município. Além disso, também foi possível verificar que os municípios com maior índice FIRJAN de desenvolvimento municipal na educação, possuem maior chance de serem plenamente eficientes.

Quanto a estrutura de regressão para o parâmetro da precisão, verificamos que os municípios com maiores índices FIRJAN de desenvolvimento municipal na renda e com maior número de consultas, apresentam respostas mais precisas, assim como a capital do estado. Ao passo que, municípios com maior proporção da população acima de 60 anos e maior média de médicos por estabelecimento de saúde, tendem a apresentar respostas menos precisas, isto é, com maior dispersão.

Dado que no Brasil os recursos disponibilizados nas esferas administrativas, em especial aos municípios, para a oferta de seus serviços são limitados, salientamos a importância no desenvolvimento de novas ferramentas ou abordagens que possibilitem a plicação dos recursos de forma mais eficiente. Conforme os resultados apresentados é possível proporcionar um melhor direcionamento de recursos destinados à atenção básica no âmbito da administração municipal, permitindo maior eficácia nos serviços oferecidos à população. Ademais, podem subsidiar políticas públicas que visem a gestão administrativa, com o propósito de buscar uma melhor aplicabilidade dos recursos destinados a esse nível de atenção.

\section{Referências}

Alves, L. A. (2012). Avaliação da eficiência na atenção básica à saúde nos municípios do estado do Espírito Santo. Tese de Doutorado, Fundação Instituto Capixada de Pesquisa em Contabilidade, Economia e Finanças-FUCAPE.

BRASIL (2004). Saúde Brasil 2004 - uma análise da situação de saúde. Ministério da Saúde. Secretaria de Vigilância em Saúde. Departamento de Análise da Situação de Saúde, Brasília.

BRASIL (2007). Assistência de Média e Alta Complexidade no SUS. Conselho Nacional de Secretários de Saúde, Brasília.

Charnes, A., Cooper, W. W., Rhodes, E. (1978). Measuring the efficiency of decision making units. European Journal of Operational Research, 2, 429-444.

Cliff, A. D., Ord, J. K. (1973). Spatial autocorrelation. London: Pion Press.

Cliff, A. D., Ord, J. K. (1981). Spatial processes: models and application. London: Pion, 12(4), 314.

Cribari-Neto, F., Pereira, T. L. (2013). Avaliação da eficiência de administrações municipais no estado de São Paulo: uma nova abordagem via modelos de regressão beta. Revista Brasileira de Biometria, 31(31), 270-294.

Cribari-Neto, F., Zeileis, A. (2010). Beta regression in R. Journal of Statistical Software, 34(2), 1-24.

Dias, R. H. (2010). Eficiência da Atenção Primária à Saúde nos municípios brasileiros. Dissertação de Mestrado, Universidade de Brasília.

Donabedian, A. (2003). An introduction to quality assurance in health care. Oxford University Press, 44(5), 655-657.

Duncan, B., Chor, D., Aquino, E., Bensenor, I., Mill, J., Schmidt, M. (2012). Doenças crônicas não transmissíveis no Brasil: prioridade para enfrentamento e investigação. Revista Saúde Pública, 46(1), 126-134.

Engstrom, S., Foldevi, M., Borquist, L. S. (2001). Is general practice effective? a systematic literature review. Scandinavian Journal of Primary Health Car, 19, 131-144.

Faria, F. P., Jannuzzi, P. M., Silva, S. J. (2008). Eficiência dos gastos municipais em saúde e educação: uma investigação através da análise envoltória no estado do Rio de Janeiro. Revista de Administração Pública, 42(1), 155-177.

Ferrari, S. L. P., Cribari-Neto, F. (2004). Beta regression for modelling rates and proportions. Journal of Applied Statistics, 31, 799-815. 
FIRJAN (2015). Índice Firjan de Desenvolvimento Municipal. Federação das Indústrias do Estado do Rio de Janeiro, URL http: //publicacoes.firjan.org.br/ifdm/2015/files/assets/common/downloads/publication.pdf.

Forrest, C. B., Starfield, B. (1996). The effect of first-contact care with primary care clinicians on ambulatory health care expenditures. Journal of Family Practice, 43(1), 40-48.

FUNASA (2006). MANUAL DE SANEAMENTO. 3. ed. rev. Fundação Nacional de Saúde, Brasília.

Greenfield, S., Komaroff, A. L., Pass, T. M., Anderson, H., Nessim, S. (1978). Efficiency and cost of primary care by nurses and physician assistants. Canadian Journal of Economics, 298(6), 305-309.

IBGE (2017). Cidades. Estimativas da População 2017. Instituto Brasileiro de Geografia e Estatística. URL ht tps : / / ci da des . ibge.gov.br/brasil/pb/panorama.

Kravitz, R. L., Duan, N., Braslow, J. (2004). Evidence-based medicine, heterogeneity of treatment effects, and the trouble with averages. The Milbank Quarterly, 82(4), 661-687.

Marinho, A. (2003). Avaliação da eficiência técnica nos serviços de saúde nos municípios do estado do Rio de Janeiro. Revista Brasileira de Economia, 57(3), 415-432.

McCullagh, P., Nelder, J. A. (1989). Generalized Linear Models. Chapman and Hall.

McFadden, D. (1974). Conditional logit analysis of qualitative choice behavior. Frontiers in Econometrics, 1, $105-142$.

Mendes, A., Marques, R. M. (2014). O financiamento da atenção básica e da estratégia saúde da família no Sistema Único de Saúde. Saúde em Debate, 38(103), 900-916.

Ospina, R., Ferrari, S. L. P. (2010). Inflated beta distributions. Statistical Papers, 51, 111-126.

Ospina, R., Ferrari, S. L. P. (2012). A general class of zero-or-one inflated beta regression models. Computational Statistics \& Data Analysis, 56, 1609-1623.

Paolino, P. (2001). Maximum likelihood estimation of models with beta-distributed dependent variables. Political Analysis, 9(1), $325-246$.

Pereira, T. L., Cribari-Neto, F. (2014). Detecting model misspecification in inflated beta regressions. Communications in Statistics: Simulation and Computation, 43, 631-656.

Pereira, T. L., Souza, T. C., Cribari-Neto, F. (2014). Uma avaliação da eficiência do gasto público nas regiões do Brasil. Ciência e Natura, 36, 23-36.

Press, W., Teukolsky, S., Vetterling, W., Flannery, B. (1992). Numerical recipes in C: The art of scientific computing. Cambridge University Press.

Queiroz, M. F. M., Silva, J. L. M., Figueiredo, J. S., Vale, F. F. R. (2013). Eficiência no gasto com saúde: uma análise nos municípios do Rio Grande do Norte. Revista Econômica do Nordeste, 44(3), 761-776.

R Core Team (2013). R: A language and Environment for Statistical Computing. R Foundation for Statistical Computing, Vienna, Austria, URL http: //www.R-project.org/.

Ramsey, J. B. (1969). Tests for specification erros in classical linear least squares regression analysis. Journal of the Royal Statistical Society, 31(2), 350-371.

Rodrigues, L. B. B., Silva, P. C. S., Peruhype, R. C., Palha, P. F., Popolin, M. P., Crispim, J. A., Pinto, J. C., Monroe, A. A., Arcêncio, R. A. (2014). A atenção primária à saúde na coordenação das redes de atenção: uma revisão integrativa. Ciência \& Saúde Coletiva, 19(2), 343-352.

Shea, S., Misra, D., Ehrlich, M. H., Field, L., Francis, C. K. (1992). Predisposing factors for severe, uncontrolled hypertension in an inner-city minority population. New England Journal of Medicine, 327(11), 776-781.

Shi, L. (1992). The relationship between primary care and life chances. Journal of Health Care for the Poor and Underserved, $3(2), 321-335$.

Silva, A. O. (2015). Regressão simplex não linear: Inferência e diagnóstico. Dissertação de Mestrado, Universidade Federal de Pernambuco. 
Simas, A. B., Barreto-Souza, W., Rocha, A. V. (2010). Improved estimators for a general class of beta regression models. Computational Statistics \& Data Analysis, 54, 348-366.

Sousa, M. C. S. (2011). Mensurando a Eficiência da Atenção Primária à Saúde em presença de outliers e fatores exógenos: uma análise em dois estágios. (Trabalho apresentado à banca examinadora como parte dos requisitos do Concurso de Professor Titular da Universidade Federal da Paraíba, na área de Economia do Setor Público). .

Souza, S. A., Oliveira, A. A., Souza, T. C., Lima, C. M. B. L. (2016). Modelagem da proporção de obesos nos Estados Unidos utilizando o modelo de regressão beta. Ciência e Natura, 38(3), 1146-1156.

Starfield, B. (1998). Primary care: balancing health needs, services, and technology. Oxford University Press.

Stasinopoulos, D. M., Rigby, R. A. (2007). Generalized additive models for location scale and shape (GAMLSS) in R. Journal of Statistical Software, 23.

Varela, P. S., Martins, G. A., Fávero, L. P. (2012). Desempenho dos municípios paulistas: uma avaliação de eficiência na atenção básica à saúde. Revista de Administração, 47(4), 624-637.

Viacava, F., Ugá, M. A. D., Porto, S., Laguardia, J., Moreira, R. S. (2012). Avaliação de Desempenho de Sistemas de Saúde: um modelo de análise. Ciência \& Saúde Coletiva, 17(4), 921-934.

Villalbi, J. R., Guarga, A., Pasarín, M. I., Gil, M., Borrell, C., Ferran, M., Cirera, E. (1999). An evaluation of the impact of primary care reform on health. Atencion Primaria, 24(8), 468-474.

Weinberger, M., Oddone, E. Z., Henderson, W. G. (1996). Does increased access to primary care reduce hospital readmissions? New England Journal of Medicine, 334(22), 1441-1447.

Woodward, C. A., Abelson, J., Tedford, S., Hutchison, B. (2004). What is important to continuity in home care? perspectives of key stakeholders. Social Science and Medicine, 58(1), 177-92.

Camila Ribeiro Silva
Universidade Federal da Paraíba, Brasil
Email: camilaribeiroufpb@hotmail.com

Participação do autor: concepção do trabalho, redação do artigo, revisão crítica e aprovação da versão final 